\title{
Challenges of single-stage pancreatoduodenectomy: how to address pancreatogastrostomies with robotic-assisted surgery
}

\author{
Lea Timmermann $^{1} \cdot$ Karl Herbert Hillebrandt $^{1,2} \cdot$ Matthäus Felsenstein $^{1,2} \cdot$ Moritz Schmelzle $^{1} \cdot$ Johann Pratschke $^{1}$. \\ Thomas Malinka ${ }^{1,3}$
}

Received: 3 August 2021 / Accepted: 21 November 2021 / Published online: 9 December 2021

(c) The Author(s) 2021

\begin{abstract}
Introduction Establishing a sufficient pancreatico-enteric anastomosis remains one of the most important challenges in open single stage pancreatoduodenectomy as they are associated with persisting morbidity and mortality. Applicability on a robotic-assisted approach, however, even increases the requirements. With this analysis we introduce a dorsal-incision-only invagination type pancreatogastrostomy (dioPG) to the field of robotic assistance having been previously proven feasible in the field of open pancreatoduodenectomy and compare initial results to the open approach by means of morbidity and mortality.

Methods An overall of 142 consecutive patients undergoing reconstruction via the novel dioPG, 38 of them in a roboticassisted and 104 in an open approach, was identified and further reviewed for perioperative parameters, complications and mortality.

Results We observed a comparable R0-resection rate $(p=0.448)$, overall complication rate $(p=0.52)$ and 30-day mortality $(p=0.71)$ in both groups. Rates of common complications, such as postoperative pancreatic fistula $(p=0.332)$, postoperative pancreatic hemorrhage ( $p=0.242$ ), insufficiency of pancreatogastrostomy ( $p=0.103)$, insufficiency of hepaticojejunostomy $(p=0.445)$ and the re-operation rate $(p=0.103)$ were comparable. The procedure time for the open approach was significantly shorter compared to the robotic-assisted approach $(p=0.024)$.

Discussion The provided anastomosis appeared applicable to a robotic-assisted setting resulting in comparable complication and mortality rates when compared to an open approach. Nevertheless, also in the field of robotic assistance establishing a predictable pancreatico-enteric anastomosis remains the most challenging aspect of modern single-stage pancreatoduodenectomy and requires expertise and experience.
\end{abstract}

Keywords Pancreatogastrostomy $\cdot$ Pancreato-enteric anastomosis $\cdot$ Pancreatoduodenectomy $\cdot$ Robotic-assisted

Reconstruction techniques following pancreatoduodenectomy (PD) are almost as old as the resection itself. In 1946, Whipple was first to perform an open single stage PD (OPD)

Thomas Malinka

thomas.malinka@charite.de

1 Department of Surgery, Charité-Universitätsmedizin Berlin, Corporate Member of Freie Universität Berlin, Humboldt-Universität zu Berlin, and Berlin Institute of Health, Humboldt-Universität zu Berlin, Berlin, Germany

2 Berlin Institute of Health, Berlin, Germany

3 Department of Surgery, Charité Campus Mitte and Charité Campus Virchow Klinikum, Charité Universitätsmedizin Berlin, Campus Virchow-Klinikum, Augustenburger Platz 1, 13353 Berlin, Germany and established restoration via pancreaticojejunostomy (PJ) [1]. Today, in the field of OPD, two main types of pancreatico-enteric anastomoses are applied. On the one hand, PJ is widely performed as a two-layered suture consisting of a duct-to-mucosa and a parenchyma-to-wall anastomosis, referred to also as the Blumgart or modified Blumgartanastomosis [2]. On the other hand, there is pancreatogastrostomy, which Waugh and Clagett initially performed in 1944 [3]. It is commonly performed as an invagination type rather than a duct-to-mucosa anastomosis. Over the past decades, generations of pancreatic surgeons have made several modifications and advocated slight advantages of either PJ or PG $[4,5]$. Comprehensive analysis, however, did not find significant differences [6, 7]. The establishment of a pancreatico-enteric anastomosis is still associated with the 
most common threats of OPD. Postoperative pancreatic fistula (POPF), postoperative pancreatic hemorrhage (PPH), or intraabdominal abscesses may result in severe morbidity and, consecutively, persistent perioperative mortality.

However, the holy grail of predictable restoration of the pancreatic remnant following OPD has not been found yet. Several risk factors for the appearance of POPF have been proposed, commonly including soft tissue texture and a narrow-calibered pancreatic duct [8]. Over the past years, minimally invasive procedures became popular in the field of pancreatic surgery. As limitations of laparoscopic techniques for such complex procedures are eminent, they remain an appropriate approach mainly for distal pancreatectomies. On the other hand, a robotic-assisted approach allows for up to seven degrees of freedom, a three-dimensional view [9] and reduces tremor transmission [10]. Therefore, it seems suitable for resection and complex reconstructions following pancreatoduodenectomy (RPD).

Nevertheless, the transferability of established techniques for pancreatico-enteric anastomoses to a robotic-assisted approach still lacks evidence. The learning curve for roboticassisted pancreatic surgery is significantly shorter than for laparoscopy [11, 12]. Several studies confirmed its safety compared to open procedures in terms of common complications [13-15]. We recently established a new dorsal-incision-only invagination type pancreatogastrostomy (dioPG) following OPD, which we now implemented for roboticassisted procedures. This study presents our first experiences with the dioPG following RPD and compares the results to our first consecutive patients undergoing dioPG following OPD for outcome parameters such as perioperative mortality and complication rates.

\section{Methods}

\section{Data collection and exclusion criteria}

We conducted a prospective single-centre observational study at our tertiary referral centre for pancreatic surgery to analyze perioperative outcome parameters of roboticassisted pancreatic surgery using the da Vinci Xi surgical system (Intuitive, Sunnyvale, CA, USA). Data of all consecutive patients who underwent $\mathrm{PD}$ with restoration of the pancreatic remnant via dioPG between October 2018 and December 2020 were collected within the CARE-Study (surgical assistance by robotic support; originally Chirurgische Assistenz durch Robotereinsatz, ethical approval code E/A4/084/17; (DRKS00017229)). Data of all consecutive patients, who underwent $\mathrm{PD}$ with restoration of the pancreatic remnant via dioPG between October 2018 and December 2020 in an open approach, were retrospectively analyzed and compared to those having undergone a robotic approach. Patients undergoing other resections, including distal and total pancreatectomies, or reconstruction via PJ or classic pancreatogastrostomy requiring a ventral gastrotomy ( $v g P G)$ were excluded from further analysis. Eleven patients undergoing RPD were excluded because reconstruction was carried out via the retrieval incision in a hybrid procedure during the program's implementation phase. We included an overall of 142 cases - 104 cases with OPD and 38 cases with RPD. We included the following data: age, sex, preoperative ASA-score, preoperative BMI, R0-resection state, operation time, overall complications, Clavien/Dindo classification, POPF, PPH, DGE, PG-insufficiency, BDA-insufficiency, surgical site infections (SSI), reoperation rate, intervention rate, in-hospital stay, 30-day mortality and 90-day readmission rate. POPF, PPH and DGE were defined and classified after the International Study Group of Pancreatic Surgery (ISGPS) classifications [16-18].

\section{Preoperative assessment and preparation}

The preoperative assessment followed a standardized schedule, including a physical examination, laboratory testing and anesthesiological evaluation. Either computed tomography with contrast agents or magnetic resonance imaging indicated resectability and confirmed given indication. In cases of underlying malignancy, an interdisciplinary tumour board evaluated each case after completed staging, including, e.g. endosonography and chest imaging.

\section{Surgical approach}

We used the da Vinci Xi surgical system for all RPDs. Electrocautery was deployed for dissection of the pancreas in both approaches. Our modified dioPG was performed as follows: after an oblique incision to the posterior gastric wall, a purse-string suture (Prolene ${ }^{\circledR} 4 / 0$ ) was placed around the cut surface without instantly tying. Afterwards, three to four mattress sutures (double armed PDS $®$ 4/0, MH1 needle) were placed through either side of the cut surface of the posterior gastric wall and the pancreatic remnant in between. Tying these mattress sutures leads to luxation of the stump through the posterior gastric wall. After tying the mattress sutures, the purse-string suture is then tied in the final step. In the case of a robotic-assisted approach, the retrieval incision served for haptic reevaluation of the implemented anastomosis. Figure 1 shows the anastomosis in progress with the untied purse-string and mattress sutures.

\section{Postoperative course}

Following an intensive care unit stay of at least one day postsurgery, patients passed to our surgical ward. The standardized course included a daily examination, laboratory testing 


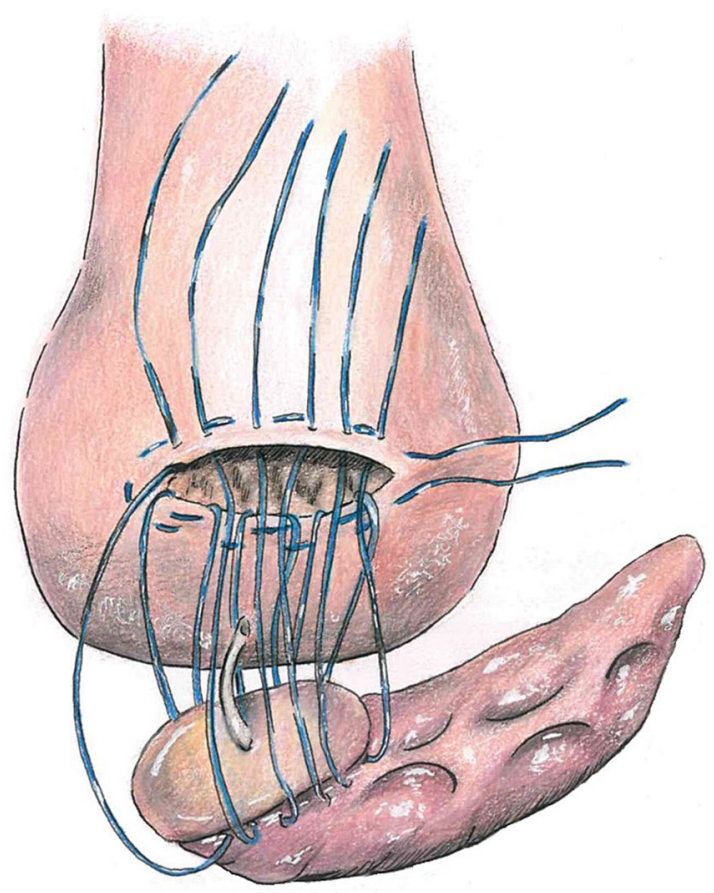

Fig. 1 dioPG in progress. Figure shows the anastomosis in progress with the untied purse-string and mattress sutures

and measuring of drainage lipase levels. If laboratory testing ruled out POPF, drainages were commonly removed on day three after surgery. An X-ray swallow study examined PG insufficiency and signs for gastric emptying disorder on day five after surgery. After removing the nasogastric tube, oral food intake was permitted.

\section{Statistics}

Data were processed using SPSS version 25.0 (IBM, Armonk, NY, USA). Two-tailed Pearson's chi-square test and Fisher's exact test were performed on categorical and ordinal scaled data, and student's t-test was performed on interval scaled data. Significance tests were two-sided, and $p<0.05$ was considered to be statistically significant.

\section{Results}

\section{Patients' characteristics}

We included an overall of 142 patients for further analysis. Thirty-eight underwent reconstruction via dioPG with a robotic-assisted approach and 104 in an open approach from October 2018 to December 2020. The patient's baseline characteristics were comparable in both groups apart from age. Patients in the RPG group were significantly younger
(61.5 years) than patients in the OPG group (68.1 years; $p=0.002$ ). Patients in the RPG group had significantly fewer malignant diseases $(p=0.002)$ compared to the OPG group. PDAC was more often an indication for surgery in the OPG group, whereas extrahepatic cholangiocellular carcinoma was more often an indication for surgery in the RPG group. In cases of underlying malignancy, T-stage was slightly higher in the OPG group, although this finding did not reach statistical significance. The tumor diameter, however, was significantly higher in the OPG group $(p=0.0002)$.

Table 1 indicates patients characteristics.

\section{Perioperative parameters}

The histopathological resection state was comparable in both groups ( $p=0.448)$, with R0-resection rates of $74.4 \%$ in the OPG group and $86.4 \%$ in the RPG group. Operation time was significantly shorter in the OPG group with $263.1 \mathrm{~min}$ (134-437 min) compared to the RPG group with $286.2 \mathrm{~min}$ (210-382 $\mathrm{min} ; p=0.024)$.

\section{Complications}

Neither the overall complication rates nor the 30-day mortality rate differed significantly in both groups. We observed an overall complication rate of $66.3 \%$ in the OPD group and $60.5 \%$ in the RPD group ( $p=0.52)$. The 30 -day mortality rate in the OPD group was $3.8 \%$ and in the RPD group 5.3\% $(p=0.71)$, whereas the overall mortality (Clavien/ Dindo 5) was $4.8 \%$ in the OPD and $7.9 \%$ in the RPD group. Three patients in the OPD group died due to cardiac arrest, one due to a severe septic shock following celiac axis occlusion and one due to hypoglycaemia. In the RPD group, one patient died due to an acute respiratory distress syndrome (ARDS), one due to severe bleeding and one due to transfusion-related acute lung insufficiency (TRALI). The rates of common complications such as POPF, PPH, DGE, SSI and insufficiency of the hepaticojejunostomy were comparable in both groups. We observed more POPF grade B in the RPD than in the OPD group $(21.1 \%$ vs $13.5 \%)$, although this finding did not show statistical significance $(p=0.103)$. The re-operation rate in the RPD group also appeared to be higher (18.4\%), although not statistically significant $(p=0.103)$, compared with the OPD group (8.7\%). Two patients in the OPD group underwent operative revision of the hepaticojejunostomy, one due to fascia dehiscence, one due to an intraabdominal haematoma, one underwent reconstruction of the celiac axis, one underwent completion pancreatectomy, one underwent splenectomy after CPR, and two underwent reoperation due to surgical site infection. Three patients in the RPD group underwent completion pancreatectomy, two underwent operative revision of the implemented PG, 
Table 1 Patients' characteristics

\begin{tabular}{|c|c|c|c|c|}
\hline Characteristics $N(\%)$ & All patients $(N=142)$ & $\mathrm{RPG}(N=38)$ & OPG $(N=104)$ & $p$ value \\
\hline $\operatorname{Sex} N(\%)$ & & & & 0.431 \\
\hline Male & $82(57.7)$ & $21(55.3)$ & $61(58.7)$ & \\
\hline Female & $60(42.3)$ & $17(44.7)$ & $43(41.3)$ & \\
\hline \multicolumn{5}{|l|}{ Age (years) } \\
\hline Mean & 66.4 & 61.5 & 68.1 & 0.002 \\
\hline Minimum & 37 & 37 & 38 & \\
\hline Maximum & 88 & 78 & 88 & \\
\hline ASA score $N(\%)$ & & & & 0.231 \\
\hline 1 & $4(3)$ & $0(0)$ & $4(4)$ & \\
\hline 2 & $67(49.6)$ & $18(51.4)$ & $49(49)$ & \\
\hline 3 & $63(46.7)$ & $16(45.7)$ & $47(47)$ & \\
\hline 4 & $1(0.7)$ & $1(2.9)$ & $0(0)$ & \\
\hline $\mathrm{BMI} N(\%)$ & & & & 0.931 \\
\hline Mean & 25.1 & 24.9 & 25.08 & \\
\hline Minimum & 17 & 17 & 17 & \\
\hline Maximum & 42 & 40 & 42 & \\
\hline Underlying malignancy $N(\%)$ & $108(76.1)$ & $22(57.9)$ & $86(82.7)$ & 0.002 \\
\hline \multicolumn{5}{|l|}{ Indication $N(\%)$} \\
\hline PDAC & $62(43.7)$ & $10(26.3)$ & $52(50)$ & \\
\hline Extrahepatic cholangiocarcinoma & $18(12.7)$ & $6(15.8)$ & $12(11.5)$ & \\
\hline Papillary carcinoma & $13(9.2)$ & $5(13.2)$ & $8(7.7)$ & \\
\hline Duodenal carcinoma & $3(2.1)$ & $1(2.6)$ & $2(1.9)$ & \\
\hline GIST & $5(3.5)$ & $0(0)$ & $5(4.8)$ & \\
\hline Other (malignant) & $7(4.9)$ & $0(0)$ & $7(6.7)$ & \\
\hline Pancreatitis & $17(12)$ & $6(15.8)$ & $11(10.6)$ & \\
\hline IPMN & $11(7.7)$ & $7(18.4)$ & $4(3.8)$ & \\
\hline Other (benign) & $6(4.2)$ & $3(7.9)$ & $3(2.9)$ & \\
\hline T-stage $N(\%)$ & & & & 0.096 \\
\hline $\mathrm{T} 1$ & $16(15.5)$ & $6(27.3)$ & $10(12.3)$ & \\
\hline $\mathrm{T} 2$ & $48(46.6)$ & $12(54.5)$ & $36(44.4)$ & \\
\hline $\mathrm{T} 3$ & $31(30.1)$ & $4(18.2)$ & $27(33.3)$ & \\
\hline $\mathrm{T} 4$ & $8(7.8)$ & $0(0)$ & $8(9.9)$ & \\
\hline Tumor diameter $(\mathrm{mm})$ & & & & 0.0002 \\
\hline Mean & 28 & 19 & 30.5 & \\
\hline Minimum & 1 & 6 & 1 & \\
\hline Maximum & 120 & 34 & 120 & \\
\hline
\end{tabular}

Table indicated baseline characteristics of patients receiving either open or robotic-assisted pancreatoduodenectomy

ASA American Society of Anesthesiologists, BMI Body Mass Index, PDAC pancreatic ductal adenocarcinoma, GIST gastrointestinal stroma tumor, IPMN intraductal papillary mucinous neoplasm

one underwent open herniotomy, and one was treated with a vacuum sealing due to SSI. Completion pancreatectomy in all cases was performed due to persisting pancreatitis and sepsis and (partial) insufficiency to the implemented PG. In two cases of the RPD group, pancreatitis appeared to be necrotizing. Operative revision of the implemented PG was performed due to partial PG-insufficiency in the early postoperative phase without pancreatitis.

Perioperative parameters are shown in Table 2.

\section{Discussion}

The implementation of a reliable pancreatico-enteric anastomosis, regardless of an open or minimally invasive approach, is the holy grail of pancreatic surgery. Life-threatening complications such as POPF, PPH and intraabdominal abscesses may occur and result in severe morbidity and mortality [7]. While the incidence of POPF decreased over the last decades, its related mortality remains at a range of around $1 \%$. 
Table 2 Perioperative parameters and complications

\begin{tabular}{|c|c|c|c|c|}
\hline Characteristics $N(\%)$ & $\begin{array}{l}\text { All patients } \\
(N=142)\end{array}$ & RPG $(N=38)$ & OPG $(N=104)$ & $p$ value \\
\hline R0 resection state $N(\%)$ & $80(76.9)$ & $19(86.4)$ & $61(74.4)$ & 0.448 \\
\hline \multicolumn{5}{|l|}{ Operation time (min) } \\
\hline Mean & 269.3 & 286.2 & 263.1 & 0.024 \\
\hline Minimum & 134 & 210 & 134 & \\
\hline Maximum & 437 & 382 & 437 & \\
\hline Overall complications $N(\%)$ & $92(64.8)$ & $23(60.5)$ & $69(66.3)$ & 0.520 \\
\hline Clavien/dindo classification $N(\%)$ & & & & 0.260 \\
\hline 0 & $49(34.5)$ & $14(36.8)$ & $35(33.7)$ & \\
\hline 1 & $9(6.3)$ & $1(2.6)$ & $8(7.7)$ & \\
\hline 2 & $14(9.9)$ & $3(7.9)$ & $11(10.6)$ & \\
\hline $3 a$ & $36(25.4)$ & $11(28.9)$ & $25(24)$ & \\
\hline $3 b$ & $7(4.9)$ & $1(2.6)$ & $6(5.8)$ & \\
\hline $4 \mathrm{a}$ & $17(12)$ & $3(7.9)$ & $14(13.5)$ & \\
\hline $4 \mathrm{~b}$ & $2(1.4)$ & $2(5.3)$ & $0(0)$ & \\
\hline 5 & $8(5.6)$ & $3(7.9)$ & $5(4.8)$ & \\
\hline $\operatorname{POPF} N(\%)$ & & & & 0.332 \\
\hline Biochemical leak & $3(2.1)$ & $0(0)$ & $3(2.9)$ & \\
\hline B & $22(15.5)$ & $8(21.1)$ & $14(13.5)$ & \\
\hline $\mathrm{C}$ & $0(0)$ & $0(0)$ & $0(0)$ & \\
\hline $\mathrm{PPH} N(\%)$ & & & & 0.242 \\
\hline A & $9(6.3)$ & $1(2.6)$ & $8(7.7)$ & \\
\hline $\mathrm{B}$ & $10(7)$ & $2(5.3)$ & $8(7.7)$ & \\
\hline $\mathrm{C}$ & $5(3.5)$ & $3(7.9)$ & $2(1.9)$ & \\
\hline $\operatorname{SSI} N(\%)$ & $13(9.2)$ & $3(7.9)$ & $10(9.6)$ & 0.753 \\
\hline DGE $N(\%)$ & $13(9.2)$ & $4(10.5)$ & $9(8.7)$ & 0.732 \\
\hline PG-insufficiency $N(\%)$ & $16(11.3)$ & $7(18.4)$ & $9(8.7)$ & 0.103 \\
\hline Insufficiency hepaticojejunostomy $N(\%)$ & $7(4.9)$ & $1(2.6)$ & $6(5.8)$ & 0.445 \\
\hline Reoperation rate $N(\%)$ & $16(11.3)$ & $7(18.4)$ & $9(8.7)$ & 0.103 \\
\hline Intervention $N(\%)$ & $53(37.3)$ & $15(39.4)$ & $38(36.5)$ & 0.250 \\
\hline 30-day mortality $N(\%)$ & $6(4.2)$ & $2(5.3)$ & $4(3.8)$ & 0.710 \\
\hline 90 -day readmission rate $N(\%)$ & $17(12)$ & $3(7.9)$ & $14(13.5)$ & 0.366 \\
\hline \multicolumn{5}{|l|}{ In-hospital stay (days) } \\
\hline Mean & 18.08 & 18.9 & 17.76 & 0.617 \\
\hline Minimum & 3 & 3 & 3 & \\
\hline Maximum & 68 & 68 & 59 & \\
\hline
\end{tabular}

Table compares perioperative complications and outcome parameters for patients receiving either open or robotic-assisted pancreatoduodenectomy

$P O P F$ postoperative pancreatic fistula, $P P H$ postoperative pancreatic hemorrhage, SSI surgical site infection, $D G E$ delayed gastric emptying, $P G$ pancreatogastrostomy
However, it may increase up to $40-50 \%$ for grade $\mathrm{C}$ fistula [7]. Tissue injuries around the cut surface, traumatic needle channels or cutting suture surfaces [19] as well as tension to the established anastomosis and blood circulation [20] may all impair the sufficiency of the applied pancreatico-enteric anastomosis. Technical challenges of robotic-assisted procedures may now add another factor. The transferability of an anastomosis, whose feasibility has already been proven in OPD, to a robotic-assisted approach is an essential point to be considered. We recently published our first experiences with robotic-assisted procedures in the field of pancreatic surgery [21]. An essential point in procedures performed on an organ with such a variable and often soft texture that instantly influences the success rate of implemented anastomoses and consecutively perioperative morbidity and mortality remains haptic feedback. Therefore, in the first RPD cases, reconstruction (hepaticojejunostomy, pancreatogastrostomy and gastroenterostomy) was performed via the retrieval incision. Although those cases were excluded from the present analysis, we consider the retrieval incision inevitable for sufficient haptic 
feedback. The evidence for pancreatico-enteric anastomoses following RPD is even less than in OPD, as only a few reports can be found [22-24]. In a published multi-centre series, minimally invasive one-row pancreaticojejunostomy appeared inferior to pancreaticojejunostomy in an open approach. The authors consider an association with the learning curve in progress during this analysis [25]. The implemented dioPG seemed to be a feasible technique in RPD setting as it allows a better exposition of the surgical site and an increased range of motion compared with the vgPG and thereby likely may decrease tissue trauma. During our first experiences with open dioPG, we additionally experienced a decrease in procedure time and rate of DGE compared to open vgPG [26]. In this current series, RPD procedures took significantly longer than OPD procedures as they include patients from our initial learning curve for robotic-assited pancreatic surgery in general. We recently showed a significant decrease in procedure time in RPD after the initial cases, that are also included in this study. The tumor diameter and the rate of underlying malignancy was significantly higher in the OPD group. This could be explained with the implementation phase of robotic assistance where tumors with larger diameter rather underwent open resection. Tumor extent, however, did not influence the technical aspects of the implemented anastomosis.

Compared to open dioPG, we saw no significant difference in perioperative complications after robotic-assisted dioPG. The rate of PG-insufficiency was higher in the RPD group, although these findings did not reach statistical significance,. As the OPD was the standard procedure over the last decades, the higher incidence of PG-insufficiency in the RPD group may have occurred due to the learning curve in progress during these first cases. Overall, the rate of PG-insufficiencies appears high in our cohort. This finding, in addition to the learning curve in progress for the RPD group of this study, might appear due to a change of management consisting of early endoscopy revealing also small insufficiencies that might have been overseen without endoscopy. We furthermore observed a slightly higher reoperation rate in the RPD group, including two patients undergoing PG-revision in the early implementation phase. The implemented anastomosis considers that an invagination type PG entirely covers the cut surface, including the proximal amount of tissue and, thereby, reduces trauma inflicted by sutures and needle channels. The implemented dioPG appears to be a technically feasible option for reconstruction following OPD and RPD. It allows an increased range of motion and a better exposition of the surgical site, especially in RPD. As this study includes cases from the initial implementation phase of robotic-assisted pancreatic surgery in our clinic, further studies are mandatory to proof our initial findings. We believe that haptic reevaluation of established anastomoses remains an essential tool and may improve the patient's safety.
This study is limited by common biases, mainly due to its retrospective character. When comparing the groups, although the RPD group was significantly younger, they were homogeneous by means of preoperative parameters (gender, BMI and ASA score). Future analyses are mandatory to further evaluate the applicability of the presented anastomosis on robotic-assisted procedures.

\section{Conclusion}

In conclusion, the implementation of a reliable pancreaticoenteric anastomosis remains an issue associated with some of the most common threats in PD. The robotic-assisted procedure furthermore increases the demands on an anastomosis to be established. With this study, we provide a technically feasible technique for the restoration of the pancreatic remnant, which is also applicable to a robotic-assisted approach.

Acknowledgements Intuitive Surgical Deutschland GmbH provided research grant for the da Vinci Xi robotic system during the study period. We greatly appreciate the contribution of Andrea Köpke, Birgül Ertugrul and Sabine Boas-Knoop to study management and data collection. $\mathrm{KH}$ and $\mathrm{MF}$ are participating in the BIH-Charité Clinician Scientist Program.

Disclosures Drs. Lea Timmermann, Karl-Herbert Hillebrandt and Matthäus Felsenstein have no conflicts of interest or financial ties to disclose. Prof. Moritz Schmelzle reports personal fees or other support apart from the present study from Merck Serono GmbH, Bayer AG, ERBE Elektromedizin GmbH, Amgen Inc., Johnson \& Johnson Medical GmbH, Takeda Pharmaceutical Limited, Olympus K.K., Medtronic $\mathrm{GmbH}$ and Intuitive. Johann Pratschke received a research grant from Intuitive Surgical Deutschland $\mathrm{GmbH}$ for the conduction of parts of the present study. Apart from this study, he reports personal fees or nonfinancial support from Johnson \& Johnson, DP Medsystems, Medtronic GmbH, Navigant, Falk Foundation, CHG Meridian, Noggo, Astellas, Promedicis, La Fourse Group, Merck Serono GmbH, Neovii, Chiesi, Med Tech, pharma-consult Peterson, I+E-research, Exafield GmbH, Eurotransplant, Intuitive, Biba Group, DSO, DTG, ESOT, ÖCK, UEG, ESOT and Fraunhofer Institut. Dr. Thomas Malinka reports on other support from Intuitive Surgical Deutschland GmbH.

Funding Open Access funding enabled and organized by Projekt DEAL. This research did not receive external funding.

\section{Declarations}

Ethical approval The study was conducted according to the Declaration of Helsinki guidelines and approved by the Institutional Review Board of Charité Universitätsmedizin Berlin (protocol code E/A4/084/17, 08.12.2017).

Informed consent Informed consent was obtained from all subjects involved in the study.

Open Access This article is licensed under a Creative Commons Attribution 4.0 International License, which permits use, sharing, 
adaptation, distribution and reproduction in any medium or format, as long as you give appropriate credit to the original author(s) and the source, provide a link to the Creative Commons licence, and indicate if changes were made. The images or other third party material in this article are included in the article's Creative Commons licence, unless indicated otherwise in a credit line to the material. If material is not included in the article's Creative Commons licence and your intended use is not permitted by statutory regulation or exceeds the permitted use, you will need to obtain permission directly from the copyright holder. To view a copy of this licence, visit http://creativecommons. org/licenses/by/4.0/.

\section{References}

1. Whipple AO (1946) Observations on radical surgery for lesions of the pancreas. Surg Gynecol Obstet 82:623-631

2. Grobmyer SR, Kooby D, Blumgart LH, Hochwald SN (2010) Novel pancreaticojejunostomy with a low rate of anastomotic failure-related complications. J Am Coll Surg 210:54-59

3. Waugh JM, Clagett OT (1946) Resection of the duodenum and head of the pancreas for carcinoma; an analysis of thirty cases. Surgery 20:224-232

4. Ricci C, Casadei R, Taffurelli G, Pacilio CA, Beltrami D, Minni $\mathrm{F}$ (2017) Is pancreaticogastrostomy safer than pancreaticojejunostomy after pancreaticoduodenectomy? A meta-regression analysis of randomized clinical trials. Pancreatology 17:805-813

5. Li Z, Wei A, Xia N, Zheng L, Yang D, Ye J, Xiong J, Hu W (2020) Blumgart anastomosis reduces the incidence of pancreatic fistula after pancreaticoduodenectomy: a systematic review and metaanalysis. Sci Rep 10:17896

6. Perivoliotis K, Sioka E, Tatsioni A, Stefanidis I, Zintzaras E, Zacharoulis D (2017) Pancreatogastrostomy versus pancreatojejunostomy: an up-to-date meta-analysis of RCTs. Int J Surg Oncol 2017:7526494

7. Pedrazzoli S (2017) Pancreatoduodenectomy (PD) and postoperative pancreatic fistula (POPF): a systematic review and analysis of the POPF-related mortality rate in 60,739 patients retrieved from the English literature published between 1990 and 2015. Medicine (Baltimore) 96:e6858

8. Chen JS, Liu G, Li TR, Chen JY, Xu QM, Guo YZ, Li M, Yang L (2019) Pancreatic fistula after pancreaticoduodenectomy: risk factors and preventive strategies. J Cancer Res Ther 15:857-863

9. Lefor AK (2019) Robotic and laparoscopic surgery of the pancreas: an historical review. BMC Biomed Eng 1:2

10. Leal Ghezzi T, Campos Corleta O (2016) 30 years of robotic surgery. World J Surg 40:2550-2557

11. Zhang T, Zhao ZM, Gao YX, Lau WY, Liu R (2019) The learning curve for a surgeon in robot-assisted laparoscopic pancreaticoduodenectomy: a retrospective study in a high-volume pancreatic center. Surg Endosc 33:2927-2933

12. Watkins AA, Kent TS, Gooding WE, Boggi U, Chalikonda S, Kendrick ML, Walsh RM, Zeh HJ 3rd, Moser AJ (2018) Multicenter outcomes of robotic reconstruction during the early learning curve for minimally-invasive pancreaticoduodenectomy. HPB (Oxford) 20:155-165

13. Magge D, Zenati M, Lutfi W, Hamad A, Zureikat AH, Zeh HJ, Hogg ME (2018) Robotic pancreatoduodenectomy at an experienced institution is not associated with an increased risk of postpancreatic hemorrhage. HPB 20:448-455

14. Zureikat AH, Moser AJ, Boone BA, Bartlett DL, Zenati M, Zeh $\mathrm{HJ}$ (2013) 250 robotic pancreatic resections safety and feasibility. Ann Surg 258:554-562

15. McMillan MT, Zureikat AH, Hogg ME, Kowalsky SJ, Zeh HJ, Sprys MH, Vollmer CM Jr (2017) A propensity score-matched analysis of robotic vs open pancreatoduodenectomy on incidence of pancreatic fistula. JAMA Surg 152:327-335

16. Wente MN, Bassi C, Dervenis C, Fingerhut A, Gouma DJ, Izbicki JR, Neoptolemos JP, Padbury RT, Sarr MG, Traverso LW, Yeo CJ, Buchler MW (2007) Delayed gastric emptying (DGE) after pancreatic surgery: a suggested definition by the International Study Group of Pancreatic Surgery (ISGPS). Surgery 142:761-768

17. Wente MN, Veit JA, Bassi C, Dervenis C, Fingerhut A, Gouma DJ, Izbicki JR, Neoptolemos JP, Padbury RT, Sarr MG, Yeo CJ, Buchler MW (2007) Postpancreatectomy hemorrhage (PPH): an International Study Group of Pancreatic Surgery (ISGPS) definition. Surgery 142:20-25

18. Bassi C, Marchegiani G, Dervenis C, Sarr M, Abu Hilal M, Adham M, Allen P, Andersson R, Asbun HJ, Besselink MG, Conlon K, Del Chiaro M, Falconi M, Fernandez-Cruz L, FernandezDel Castillo C, Fingerhut A, Friess H, Gouma DJ, Hackert T, Izbicki J, Lillemoe KD, Neoptolemos JP, Olah A, Schulick R, Shrikhande SV, Takada T, Takaori K, Traverso W, Vollmer CR, Wolfgang CL, Yeo CJ, Salvia R, Buchler M, International Study Group on Pancreatic S (2017) The 2016 update of the International Study Group (ISGPS) definition and grading of postoperative pancreatic fistula: 11 years after. Surgery 161:584-591

19. Kawaida H, Kono H, Hosomura N, Amemiya H, Itakura J, Fujii H, Ichikawa D (2019) Surgical techniques and postoperative management to prevent postoperative pancreatic fistula after pancreatic surgery. World J Gastroenterol 25:3722-3737

20. Ke Z, Cui J, Hu N, Yang Z, Chen H, Hu J, Wang C, Wu H, Nie X, Xiong J (2018) Risk factors for postoperative pancreatic fistula: analysis of 170 consecutive cases of pancreaticoduodenectomy based on the updated ISGPS classification and grading system. Medicine (Baltimore) 97:e12151

21. Timmermann L, Biebl M, Schmelzle M, Bahra M, Malinka T, Pratschke J (2021) Implementation of robotic assistance in pancreatic surgery: experiences from the First 101 Consecutive Cases. J Clin Med 10

22. Giulianotti PC, Gonzalez-Heredia R, Esposito S, Masrur M, Gangemi A, Bianco FM (2018) Trans-gastric pancreaticogastrostomy reconstruction after pylorus-preserving robotic Whipple: a proposal for a standardized technique. Surg Endosc 32:2169-2174

23. Menonna F, Napoli N, Kauffmann EF, Iacopi S, Gianfaldoni C, Martinelli C, Amorese G, Vistoli F, Boggi U (2020) Additional modifications to the Blumgart pancreaticojejunostomy: results of a propensity score-matched analysis versus Cattel-Warren pancreaticojejunostomy. Surgery

24. Wang SE, Shyr BU, Chen SC, Shyr YM (2018) Comparison between robotic and open pancreaticoduodenectomy with modified Blumgart pancreaticojejunostomy: a propensity scorematched study. Surgery 164:1162-1167

25. Klompmaker S, van Hilst J, Wellner UF, Busch OR, Coratti A, D'Hondt M, Dokmak S, Festen S, Kerem M, Khatkov I, Lips DJ, Lombardo C, Luyer M, Manzoni A, Molenaar IQ, Rosso E, SaintMarc O, Vansteenkiste F, Wittel UA, Bonsing B, Groot Koerkamp B, Abu Hilal M, Fuks D, Poves I, Keck T, Boggi U, Besselink MG, European consortium on Minimally Invasive Pancreatic S (2020) Outcomes after minimally-invasive versus open pancreatoduodenectomy: a Pan-European Propensity Score Matched Study. Ann Surg 271:356-363

26. Timmermann L, Bahra M, Pratschke J, Malinka T (2021) Development of a novel dorsal incision only invagination type pancreatogastrostomy (Charite-PG) following open pancreaticoduodenectomy-a single centre experience. J Clin Med 10

Publisher's Note Springer Nature remains neutral with regard to jurisdictional claims in published maps and institutional affiliations. 